\title{
PRESENTACIÓN
}

\section{DE LA REFLEXIÓN A PARTIR DE PROYECTOS Y EVENTOS EN VIVIENDA SOSTENIBLE Y ADAPTADA: APUNTE DE LOS CONGRESOS SHUR 2015 Y GREENCITIES '14}

\author{
María Luisa Gómez Jiménez \\ Directora
}

Sirvan estas líneas para iniciar la presentación del número 2 de la Revista WPSRISHUR. Una publicación que incorpora desde una visión interdisciplinar el análisis integrado de aspectos urbanísticos y edificatorios desde la proyección no sólo del derecho sino de las nuevas tecnologías, la ingeniería la arquitectura y la proyección práctica de los profesionales de la vivienda y la rehabilitación Sostenible.

En esta segunda ocasión, resultaba de especial interés destacar la significativa oportunidad de reflexionar en el impacto de las nuevas tecnologías en la vivienda y rehabilitación sostenible. La razón para ello trae causa de dos foros convergentes. El primero fue el Congreso Greencities, que tuvo lugar en Málaga en el mes de Octubre de 2014 y que reunió más de 2000 profesionales del sector de la vivienda y la construcción, y el Congreso SHUR Smart Homes and Urban Renewal, que primero en su categoría, incorporó la proyección tecnológica de la ingeniería y la arquitectura en la domótica y sostenibilidad en la vivienda.

Pues bien la confluencia de ambos eventos, ha motivado que este número pueda incorporar el reflejo de aportes que se realizaron y presentaron oportunamente en ambos foros, y que complementan las publicaciones realizadas hasta la fecha ya desde la organización de Greencities Málaga. Es por ello este un número ecléctico, porque aborda cuestiones en apariencia disimiles pero a la par interesantes. Así, comenzamos el número con el aporte de la profesora de arquitectura D. Susana García Bujalance, que en una aproximación innovadora nos habla del territorio como tejido y como entramado en el que todo se sucede con la limitación que el espacio proporciona pero con la exultante oportunidad de mejora que incorpora. Nos habla la Profesora Bujalance de las Cartografías del Territorio y nos sitúa en la ciudad de Málaga, con la certera apreciación diagnóstica de los efectos inmediatos de la crisis inmobiliaria y su recuperación que abogue por una diferente aproximación integrada al territorio su análisis y proyección desde lo histórico a lo actual.

WPS RI-SHUR, no2, 2015, vol.1, ISSN: 2387-1768 


\title{
WPSReview International on Sustainable
}

\author{
Housing and Urban Renewal (RI-SHUR)
}

Pues bien es preciso un enfoque amplio, multidisciplinar para atender a las cuestiones sociales que se dibujan con carácter permanente en el escenario jurídico. Por ello el segundo aporte aunque pudiera resultar alejado en temática no lo es, y ahí radica su innovadora contribución. No podía ser de otra manera el Prof. Dr. Martín Bassols Coma, Catedrático de Derecho Administrativo de la Universidad de Alcalá, Letrado de las Cortes y Presidente de la Asociación Española de Derecho Urbanístico, nos ilustra con precisión y brillantez sobre una problemática de enorme actualidad como sea los medios de defensa de los operadores económicos ante la libertad de establecimiento y circulación en el marco de la Ley de Garantía de Unidad de Mercado. Pues las reflexiones del Prof. Bassols Coma son especialmente de interés para el examen de la actuación de los operadores económicos en el mercado, integrando en los mismos todos los que han permitido la definición de políticas de urbanísticas y edificación. Esto es, con plena aplicabilidad al ámbito del mercado de la Vivienda. Es quizás el único aporte plenamente jurídico en este volumen segundo, pero su relevancia amerita su presencia sin que ello desmerezca nada a la proyección profesional y práctica de las presentaciones en los foros que reseñamos, sino más bien al contrario permita no perder la visión interdisciplinar con la que aproximaremos el estudio de los temas en este y números sucesivos.

Así, completa el bloque de las reflexiones en materia de accesibilidad a la Vivienda un trabajo que fue presentado en el seno del Foro Greencities y que destaca por su proyección e integración participativa, nos referimos a la contribución de la Profesora D. María López de Asiaín Alberich, de la Universidad de Sevilla. La Profesora López de Asiaín, nos presenta el proyecto EUOBs, cuya andadura ha permitido poner en valor el empoderamiento de la población en la toma de decisiones relacionadas con la sostenibilidad de vivienda y el impacto de la misma en la calidad de vida de los ciudadanos. El proyecto que ha sido financiado por la Consejería de Economía, Innovación, Ciencia y Empleo en 2014, ha sido desarrollado en un consorcio con las Universidades de Málaga, Granada, Sevilla y Habitec, Fundación sin ánimo de lucro que viene a coordinarlo. El aporte que presentamos ha sido elaborado por los expertos Salas Mendoza y Cano Ruano., y presentado además en el Congreso SHUR, el pasado 24 de Junio.

En sucesión de continuidad con el anterior, y respecto de las propuestas en rehabilitación de Vivienda destacan dos contribuciones que vienen a abordar temáticas desde la proyección institucional de la Administración Regional de un lado y desde la Administración Local de otro. Nos referimos a la contribución que realiza el Instituto Valenciano de la Edificación en el trabajo titulado, "Iniciativa Rehab calidad en tu vivienda, como involucrar al ciudadano en el proceso de mejora de calidad de la

WPS RI-SHUR, nำ2, 2015, vol.1, ISSN: 2387-1768 


\title{
WPSReview International on Sustainable \\ Housing and Urban Renewal (RI-SHUR)
}

vivienda", pues bien esta trabajo que ha sido presentado en el foro Greencities en octubre de 2014, viene a incorporar la experiencia el Instituto Valenciano de la Edificación en rehabilitación de vivienda. La presentación completa y sistemática aborda aspectos claves que implicación y participación de los administrados en la mejora de su calidad de vida, en esta ocasión desde la iniciativa pública, y las instituciones administrativas, sin dejar de situar al ciudadano en el centro de la misma.

Por último este número cierra las contribuciones sobre rehabilitación con la propuesta que emana del Ayuntamiento de Fuengirola, y que fue presentada en el congreso SHUR, sobre mecanismos de gestión eficiente del alumbrado público, a través del uso de nuevas tecnologías. El aporte que emana en esta ocasión de los Profesores Hermoso Orzáez, a la sazón Ingeniero Municipal del Ayuntamiento de Fuengirola y Profesor del Área de Proyectos en la universidad de Jaén; D. José Ramón Andrés Díaz, Profesor de la Universidad de Málaga y D. Guillermo Redrado, Vicepresidente de operaciones de ATP, lluminación.

Ciérrese de esta manera el círculo que se inicia en el primer aporte del volumen desde la cartografía del territorio malagueño a la previsión de sostenibilidad ambiental y energética en el municipio de Fuengirola, buscándose con ello una proyección de lo internacional en lo local, y de lo nacional en lo inmediato que conoce el ciudadano.

Pero no demoro más con estas breves líneas lo que el lector seguro desea, eso es, sin más adentrarse en la lectura del nuevo volumen que en este mes de septiembre se presenta de nuestra publicación periódica WPS- RI-SHUR.

En Málaga a 10 de Septiembre de 2015

\author{
María Luisa Gómez Jiménez \\ Directora
}

WPS RI-SHUR, nำ2, 2015, vol.1, ISSN: 2387-1768 